\title{
Investigación médica en seres humanos en Alemania: Reglas legales y profesionales y cuestiones éticas debatibles*
}

\author{
Monika Bobbert, Universidad de Heidelberg (Alemania)
}

Recibido: 12 de julio del 2009 / Aprobado: 31 de agosto del 2009

Este artículo explora la situación de la investigación en seres humanos a raíz de algunas modificaciones ocurridas en Alemania en los últimos diez años. El estudio de los tres tipos de argumentaciones hechas sobre estas investigaciones, la deontológica, la contractualista y la utilitarista demostrarían que se está produciendo una mayor valoración a la investigación en medicina y menor consideración a los riesgos y dolencias de los pacientes sujetos de experimentación. La autora sostiene la importancia no solo de utilizar el criterio del consentimiento informado que podría ser, en el fondo, limitante de la autonomía, sino de otros criterios éticos que defiendan el bienestar y las ventajas de los pacientes experimentales.

bioética / Alemania

\section{Human medical research in Germany: Legal and professional rules and} debatable ethics topics

This paper analyzes the situation of human research in light of some changes that have taken place in Germany in the past ten years. The study of the three main arguments regarding human experimental research, namely, the deontological, the contractualist and the utilitarian reveal that there is a tendency to place higher value on the research and less consideration for the risks and well-being of the experimental subjects. The author claims for an inclusion of several ethical criteria in addition to the solely informed consent which, in many cases of experimental research, may curtail the patient's autonomy, and argues for the importance of the well-being and advantages of the subjects.

bioethics / Germany

Correo electrónico: Monika.Bobbert@histmed.uni-heidelberg.de

* Traducción del alemán de Ramón León. 


\section{INVESTIGACIONES EN SERES HUMANOS en la República Federal de Alemania}

Todas las reglamentaciones legales vigentes en Alemania destacan la protección de la persona que participa en experimentos en medicina como la prioridad. Esto, ciertamente, concuerda con el objetivo de la práctica médica: promover la salud y el bienestar del paciente individual. Pero un médico que investiga se encuentra, por el contrario, en un dilema, dado que la investigación tiene como meta la generación de nuevos conocimientos o el bienestar de futuros pacientes. Este conflicto entre el bienestar del paciente individual y los intereses de la ciencia médica es en realidad insoluble y demanda, por eso, reglas legales y lineamientos éticos (Mieth, 2002).

La Constitución alemana se basa en imprescriptibles derechos humanos, categorialmente válidos, que no pueden ser transgredidos: por ejemplo el derecho a la vida y a la integridad física, así como el derecho a la libertad (Grundgesetz für die Bundesrepublik [1949]). De esto se deriva el derecho al cuidado de la salud y a la aprobación informada y voluntaria por parte del paciente, en los casos en que sea necesaria la intervención en su integridad física o psicológica. Asimismo, la ley asegura el derecho a la autodeterminación informada y al cuidado de las informaciones referidas a la persona y a su salud (Bundesdatenschutzgesetz,
1978). Además, algunas leyes específicas, la Gesetz über den Verkehr mit Arzneimitteln (2005) y la Gesetz über Medizinprodukte (2002), son leyes que regulan el ensayo de nuevos medicamentos o productos médicos en los seres humanos. Así, la investigación en seres humanos debe ser permitida solo cuando los nuevos conocimientos no puedan ser obtenidos de otra manera por ejemplo, a través de la experimentación en animales - y con la condición de que los riesgos previsibles y las eventuales molestias de las personas con las cuales se lleva a cabo la investigación sean "médicamente aceptables" en relación con los probables beneficios del proyecto de investigación.

Recomendaciones éticas de naturaleza profesional, como las directivas de la Cámara Federal de Médicos (Bundesärztekammer) o la Declaración de Helsinki del 2008, una recomendación internacional de la Federación Mundial de Médicos para la investigación con seres humanos ofrecen criterios valorativos y planteamientos adicionales (World Medical Association, 2008).

En Alemania el principal requisito formal que debe satisfacer un proyecto de investigación es su aprobación a través de una comisión de ética. En el caso de ensayos de medicamentos o de evaluación de productos médicos la comisión debe dar un voto positivo en aspectos médicos, legales y metodoló- 
gicos. En todas las otras formas de la investigación en seres humanos la comisión ética tiene solo estatus de asesora. De acuerdo con una sugerencia de la Declaración de Helsinki de 1975 deberían fundarse comisiones éticas interdisciplinarias a fin de dictaminar sobre proyectos de investigación médica en seres humanos.

La Cámara Federal de Médicos implementó la recomendación de la Federación Médica Mundial a mediados de la década de 1980. Las comisiones de ética están constituidas por alrededor de 10 miembros, de los cuales la gran mayoría proviene de la medicina. Pero también otras disciplinas, como el Derecho, la Biometría, la Teología y la Filosofía, están parcialmente representadas. Esta comisión ética de investigación forma parte en unos casos de una Facultad de Medicina y la integran sobre todo médicos con actividad docente, o está ubicada en las así llamadas cámaras de cada uno de los estados alemanes. En este caso, los médicos de un determinado estado federal alemán conforman la mayoría de sus integrantes. Esta estructura de la comisión es considerada como una forma de autorregulación y autocontrol profesionales.

Las comisiones éticas de investigación tienen así una función clave y su conformación, forma de trabajo y criterios de decisión son determinantes para su trabajo independiente y competente. Sin embargo, en Alemania siempre surgen voces críticas sobre el particular, porque el proceso mediante el cual se toman decisiones no está claramente reglamentado (¿es requisito el voto de mayoría o no lo es?, ¿cómo se debe proceder cuando hay un dictamen en minoría?), porque los criterios de decisión varían (por ejemplo, unos están más orientados al plano legal en tanto que otros lo están más al ético) y porque los miembros de la comisión muchas veces son (cercanos o lejanos) colegas de quienes presentan el proyecto de investigación y, debido a ello, su independencia puede encontrarse mediatizada. Asimismo, se critica que solo se suele evaluar el proyecto en sus comienzos, pero que no hay ninguna supervisión en el transcurso y en el final de este.

Por último, debe señalarse que el financiamiento de las comisiones de ética no está reglamentado. Estas comisiones se financian en parte con subvenciones de facultades médicas o de las cámaras médicas federales, esto es entidades de derecho público, pero también en una buena medida a través de la evaluación de solicitudes para la investigación de medicamentos y productos médicos. Esto ha dado lugar a una competencia entre las diferentes comisiones, pues existe - bajo ciertas condiciones - algún interés económico para no derivar, por ejemplo, solicitudes de investigación provenientes de la industria farmacéutica a otras comisiones que pudieran ser menos rigurosas en los 
aspectos de valoración legal y crítica, perdiendo así la posibilidad de eventuales ingresos.

\section{ALGUNAS MODIFICACIONES EN LAS REGLAMENTACIONES LEGALES $Y$ PROFESIONALES EN LOS ÚLTIMOS DIEZ AÑOS}

En los últimos diez años se han producido modificaciones en las recomendaciones europeas (Europäisches Parlament, 2001), en las normas legales en Alemania (Gesetz über den Verkehr mit Arzneimiteln, 2004; Rothaar, 2006) y en los códigos de ética. Estos cambios deben ser valorados críticamente, porque han debilitado la protección del sujeto experimental. En lo que sigue se presentarán las tres modificaciones centrales.

\section{Supresión de la diferencia entre la investigación terapéutica y la no terapéutica}

La diferencia entre la investigación terapéutica y la no terapéutica, tal como fue incluida en la Declaración de Helsinki pero también en leyes nacionales, ha sido en gran medida abandonada. Es desde el punto de vista de la ética algo muy criticable. Pues, aunque en la práctica la frontera entre una y otra no siempre puede ser precisada con claridad, para la valoración ética constituye una gran diferencia si los pacientes que participan en un proyecto de investigación pueden tener la esperanza de alguna mejoría de su propio estado de salud o si, desde un inicio, está claro que solo los futuros pacientes o la sociedad podrán disfrutar de los potenciales beneficios de ella.

El hecho de que algunos proyectos de investigación no puedan ser clasificados de manera clara como proyectos terapéuticos o no terapéuticos (en parte porque una nueva forma de tratamiento que es evaluada posiblemente no llegue a tener utilidad alguna o solo una utilidad terapéutica muy pequeña), no puede ser el fundamento para abandonar o descuidar la interrogante ética acerca de la determinación de quién será el beneficiario (o eventualmente el perjudicado) con el estudio que se pretende llevar cabo. Más bien, la respuesta clara a esa pregunta es la base para una adecuada valoración médica, tanto objetiva como ética. La valoración ética de un proyecto de investigación clínica dependerá de si los pacientes que participan en él pueden esperar algún beneficio potencial para ellos, o si solo las futuras generaciones serán las que disfruten de los beneficios provenientes de sus resultados. Los pacientes que padecen de una enfermedad incurable y que participan como probandos en un proyecto de investigación acerca de un nuevo tratamiento porque tienen la esperanza de una mejoría de su propio estado, bajo determinadas circunstancias podrán tolerar mayores molestias y asumir riesgos más eleva- 
dos dado que la enfermedad supone para ellos una severa limitación en su calidad de vida.

$\mathrm{Si}$, empero, los médicos en su rol de investigadores solicitan a sus pacientes o a determinadas personas que participen en un proyecto de investigación no terapéutica, los pacientes saben que asumen de manera unilateral solo molestias y riesgos en beneficio de otros. Por ello, en la investigación no terapéutica los potenciales riesgos y molestias tienen que ser mínimos, pues no se puede justificar la permanente exposición de la salud y el bienestar de una persona a riesgos y potenciales peligros.

El cuidado de la persona experimental ante los riesgos y peligros inducidos por la investigación tendría todavía que ser mayor si los médicos no pueden contar con personas sanas en un proyecto de investigación no terapéutica, de modo tal que solo sus pacientes pueden ser los sujetos experimentales. Por un lado, los pacientes están ya, debido a su enfermedad, debilitados y expuestos a peligros; del otro, se encuentran en una situación de dependencia con respecto a sus médicos tratantes y confian en que estos les prescribirán el mejor tratamiento.

Pero, además, la relación médicopaciente podría también ser el motivo por el cual vacilen en decir no, es decir en negarse a participar en un proyecto de investigación, si su médico tratante le sugiere formar parte de este en calidad de probando.
En general, la clarificación de la pregunta acerca de si los sujetos experimentales o pacientes tienen que esperar ventajas o daños, y en qué forma, debe estar en el primer lugar, debido al necesario cuidado y a los derechos que posee todo sujeto experimental. El médico investigador debe informar seria y responsablemente a sus pacientes, ofreciéndoles una respuesta lo más clara posible acerca de cómo y en qué medida podrían esperar algunas ventajas de su participación en un proyecto de investigación.

Sin embargo, si la diferenciación sistemática entre la investigación terapéutica y la no terapéutica está ausente, y por tanto también lo está la preocupación por determinar la naturaleza del proyecto, entonces los objetivos pueden ser poco transparentes, tornando sumamente difícil la valoración ética para todos los participantes.

\section{Investigación no terapéutica con grupos especiales de riesgo}

En los últimos años es posible observar que han disminuido el cuidado y la preocupación por los grupos que se encuentran en particular estado de riesgo. Esto se evidencia en el ámbito europeo, por ejemplo en la llamada Convención de Bioética de 1997 (Europarat, 1997), o en los lineamientos acerca de la aplicación de una "Buena Práctica Clínica" (GCP) (Europäisches Parlament und der Rat der Europäischen 
Union, 2001). Ambas aprueban, en determinadas circunstancias, la investigación en niños que no traerá ningún beneficio para ellos. La Convención de Bioética permite como excepción también la investigación no terapéutica en pacientes que no están en condiciones de dar su aprobación para participar en ella: pacientes con enfermedades neurológicas o psiquiátricas o personas con retardo mental.

Que estos grupos de personas particularmente expuestas a riesgos pueden ser sistemáticamente objeto de investigación, lo demuestran además algunas nuevas reglamentaciones nacionales. Así, por ejemplo, en Canadá y en Holanda hay una reglamentación especial para la investigación en pacientes que no están en condiciones de dar su aceptación (van Dijk et al., 2003; Law of Medical Research Council of Canada, 1998).

Alemania no ha ratificado hasta ahora la Convención de Bioética de la Unión Europea, porque en ella está permitida la investigación no terapéutica en pacientes imposibilitados de dar su aprobación debido a su precario estado de salud (por ejemplo, personas con demencia u otro tipo de severos problemas psicológicos). Al mismo tiempo, con motivo de la renovación de las leyes de medicamentos en el año 2005, a iniciativa de la Reglamentación Europea para la buena práctica clínica, se suscitó un intenso debate político, que concluyó permitiendo la investiga- ción no terapéutica en menores de edad bajo determinadas condiciones.

\section{Diseño de placebo en presencia de una terapia efectiva}

Hasta el año 2000 la Declaración de Helsinki, el código internacional de la Federación Médica Mundial, excluyó los grupos placebo, es decir los seudotratamientos, en la medida en que había una terapia estándar probada. Por cierto, se permitían los grupos placebo en un diseño de investigación cuando ningún tratamiento efectivo existía y se debía investigar si una nueva terapia era más eficaz que no llevar a cabo tratamiento alguno, o un seudotratamiento. Un comentario en una nota de pie de página de la Declaración de Helsinki del 2002 presentó un cambio considerable de posición. Esta adición permitía, bajo determinadas circunstancias, evaluar nuevas terapias con respecto a un placebo, aunque exista un tratamiento estándar; esto es, cuando las condiciones científicas hacían necesaria tal investigación o cuando no era de esperar para el paciente algún riesgo o daño irreversible. En especial la relación de las dos condiciones a través de una " $o$ " fue crítica, dado que de esta manera el bienestar de la persona quedaba subordinado a los logros científi$\cos$ en materia de conocimiento. La nueva versión de la Declaración del 2008 confirmó - a pesar de la crítica internacional existente- este modo de ver las cosas. Solo se agregó: "extreme 
care must be taken to avoid abuse of this option".

Con la fórmula best current proven intervention la Declaración determinó el mejor estándar como comparación para estudios clínicos. Sin embargo, hay regiones en el mundo en las cuales no se dispone de excelentes estándares médicos. Queda librada a la interpretación esta frase, si con la determinación a la mejor intervención asegurada en países con deficientes sistemas de salud el segundo mejor estándar debe ser tomado en cuenta o igual un grupo placebo puede considerarse como apropiado.

En general, en la Declaración de Helsinki esta frase significa la legitimación del diseño experimental con un grupo placebo que a lo largo de la duración del experimento no recibe ni el tratamiento efectivo ni el nuevo tratamiento, que podría ser potencialmente efectivo.

\section{TRES ARGUMENTACIONES ÉTICAS}

Las modificaciones antes señaladas son indicadores de la tendencia a conceder mayor significado a la ventaja potencial de la generación de nuevos conocimientos y de la investigación terapéutica para futuros enfermos que a la consideración de los malestares y riesgos del sujeto de ensayo individual.

Esto puede llevar a una limitación de los derechos individuales. En el lenguaje técnico de las teorías y las argumen- taciones éticas es posible decir que se registra un cambio de los siguientes tres tipos de argumentos: argumentaciones deontológicas basadas en el derecho, argumentaciones contractualistas y argumentaciones utilitarias (teleológicas).

\section{Argumentaciones basadas en el derecho}

Los derechos humanos individuales se basan en el principio de que cada persona tiene un valor en sí misma y no puede ser instrumentalizada por otros (tradición kantiana). Además, hay una jerarquía de derechos morales individuales: el derecho al cuidado de la vida y el del acceso a un buen sistema de salud son los más elevados, así como el derecho a la integridad física y psíquica. Al cuidado de estos derechos individuales debe dedicársele toda suerte de atenciones. Los derechos individuales fundamentales tienen un valor categórico, esto es que son independientes del valor que los demás conceden a un individuo.

Los problemas de tal argumentación son, entre otros, los siguientes: los derechos fundamentales deben ser interpretados y aplicados. En caso de un conflicto entre los derechos básicos de varios individuos, se necesitan reglas de valoración adicionales y la consideración del contexto específico. Por esto, la argumentación con derechos humanos debe también ser enriquecida con otros criterios éticos, y 
estos deben ser debidamente fundamentados.

Un problema adicional consiste en la implementación de los derechos humanos. Aunque las normas de los derechos humanos tienen una validez irrestricta, su cumplimiento no siempre está garantizado en la práctica. Muchas veces son posibles solo pequeños pasos en la dirección correcta, y en esos casos los derechos humanos sirven solo como "ideal correctivo". Sin embargo, una práctica inhumana no pone en duda la validez de la norma moral. Más bien esas normas, en el caso de transgresiones de los derechos humanos, exigen una modificación de la praxis.

\section{Argumentaciones contractualistas}

En las teorías contractualistas los actores estratégico-racionales encuentran un consenso. Ninguno de los participantes está obligado a suscribir un contrato. Determinados contenidos o normas del acuerdo se basan en la concordancia de los socios sobre el particular. $\mathrm{Su}$ contenido no es por tanto criticable desde afuera, sino que depende del criterio de los miembros del contrato. En esta argumentación el acuerdo informado del partner en el contrato o de la persona que participa en el experimento se halla en el centro de todas las consideraciones.

Esta es también la única justificación para que los contenidos o normas acordados tengan validez. La autoridad moral descansa por ello en los respectivos participantes y en el acuerdo al que han llegado.

Desde el punto de vista crítico, es cuestionable la suposición de que las normas éticas y las valoraciones solo puedan ser legitimadas a través de acuerdos fácticos o teóricos entre individuos con intereses propios. Posiciones controversiales o intereses que no pueden conciliarse no pueden explicarse a través de este enfoque. La perspectiva moral se acomoda a los acuerdos, a los cuales no puede agregarse ninguna perspectiva crítica adicional.

En la investigación médica en seres humanos esta argumentación se caracteriza por un marcado entusiasmo en lo que se refiere a la aprobación informada de la persona para participar en un proyecto de investigación. Un problema de tal argumentación consiste en que el interlocutor informado y autónomo constituye en realidad un requisito previo. Ahora bien: ¿cómo controlar sin embargo la asimetría en lo que se refiere a poder e información existente entre el médico y el paciente, o entre el investigador como experto y el probando como lego? Es difícil saber en qué medida los pacientes que participan en un estudio clínico están en verdad informados y realmente participan de manera voluntaria en un proyecto de investigación, cuando se solicita la colaboración de ellos en el marco de un tratamiento y ninguna otra regla ética es considerada aparte de la del derecho 
a la propia determinación. Pues otras normas éticas, como el cuidado de la vida y de la salud de la persona, la valoración ética del objetivo de investigación y la valoración de las ventajas y desventajas para los afectados, no están incluidas en este enfoque contractual.

\section{Argumentaciones utilitarias}

La norma máxima en las teorías utilitaristas es el principio de la maximización de la utilidad común de todos los participantes. La definición de "utilidad" varía y puede dar lugar a numerosas discusiones. Así, por ejemplo, la recuperación del deseo de seguir viviendo y la evitación del dolor cuentan como utilidad, pero también los años de vida ganados o la conservación de facultades cognitivas, y aun es posible expresar esa utilidad en categorías financieras cuantificables.

Problemas de esta argumentación son, entre otros, los siguientes: la justificación de la maximización del provecho o utilidad no es suficiente cuando se argumenta solo empíricamente. Esto es, la suposición de que la mayoría de personas solo busca el placer y evitar el dolor puede ser cuestionada en su validez. De otro lado, se pasa de largo frente a la diferencia sistemática entre ética y moral y entre el ser y el deber. Aquello que desde el ángulo ético es bueno y correcto no se puede determinar sencillamente a través de lo empírico y de los hechos humanos fácticos.
También la determinación del contenido de la utilidad o provecho es siempre cuestionable, porque depende de valoraciones sociales y de definiciones establecidas por la mayoría. En general, una argumentación utilitaria corre el peligro de que determinados individuos con necesidades básicas sean dejados de lado porque ellos no significan nada ni tienen peso alguno en la suma de las utilidades totales. En otras palabras, los derechos humanos básicos no están garantizados con este enfoque. En la investigación en seres humanos existe la tendencia a considerar como muy elevada la utilidad potencial de un proyecto de investigación — por ejemplo, a través del desarrollo de una posibilidad de tratamiento para una enfermedad hasta entonces incurable - $y$, basado en la posibilidad de éxito, justificar grandes riesgos y malestares a un grupo de personas que sirven como sujetos experimentales de esa investigación.

\section{Prioridades MODIFICADAS EN LAS ARGUMENTACIONES COMO PROBLEMA ÉTICO}

Ciertamente, en la investigación en seres humanos siempre hay una mezcla de las tres argumentaciones previamente mencionadas. Esto caracteriza este campo de la ética aplicada, pues la investigación en seres humanos es siempre un compromiso entre el cuidado individual y el provecho de la terapia y una utilidad común. 
Sin embargo, en Alemania y en las versiones de la Declaración de Helsinki tuvo prioridad durante décadas la argumentación basada en los derechos individuales, la deontológica. Las dos otras argumentaciones tuvieron un rol subordinado. Desde hace algunos años, sin embargo, aparecen con más frecuencia los argumentos de la contractualización o del utilitarismo en primer lugar.

\section{Graves efectos colaterales para pacientes en la investigación clínica}

La argumentación contractualista ocupa el primer lugar cuando debe ser probado un medicamento para pacientes gravemente enfermos, a pesar de que ocasione severos efectos colaterales o pueda traer consigo el riesgo de un acortamiento de la vida. La pregunta acerca de si un proyecto debe ser llevado a cabo es respondida con el hecho de que se cuenta con la aprobación del paciente, que está, además, debidamente informado.

Se extraña en estas circunstancias una valoración previa por parte del investigador acerca de si el medicamento debe en absoluto ser verificado. Ciertamente, se trataría en este caso de investigación terapéutica, por lo tanto existiría la posibilidad de que algunas personas que participaran en el proyecto pudieran beneficiarse. Pero el interrogante acerca de si, por ejemplo, una prolongación de la vida de unos 10 días jus- tifica la administración de un medicamento que puede traer consigo graves consecuencias colaterales, es algo que el investigador debe contestar. Si todas estas preguntas éticas quedan sencilla y llanamente "solucionadas" por el hecho de que la persona está informada y se le deja a ella la posibilidad de decidir su participación en el experimento, entonces la teoría contractualista es la que está predominando.

Desde el punto de vista ético esto es problemático, porque en la investigación clínica el paciente debe ser invitado para participar en un ensayo, y él se halla en una situación de dependencia del médico tratante y del hospital en el que se encuentra. Y en parte, además, en una situación sin salida en la cual debe recurrir a cualquier posibilidad terapéutica, si bien no existe ninguna posibilidad de curación.

Sin embargo, referirse sencillamente al derecho de cada persona a tomar decisiones puede ser problemático cuando se trata de personas sanas. Pues ellas, como legos, no siempre están en condiciones de valorar correctamente el aspecto científico del experimento, sino que deben guiarse por las informaciones y las valoraciones del riesgo que llevan a cabo. Una simple argumentación de corte contractualista no basta en la investigación, puesto que el médico siempre debe garantizar que la vida de la persona, de acuerdo con su valoración, no sea dañada, o que ella no corra algún riesgo que ocasione perjui- 
cios permanentes a su salud. Los investigadores experimentales responsables deben también tomar una decisión previa de corte técnico y ético, la cual debe ser formulada explícitamente. Si por medio de un desplazamiento del peso de los argumentos, son la información y aprobación del sujeto experimental lo decisivo, entonces se asigna a la persona demasiada responsabilidad. En lugar de esto es necesario que el investigador lleve a cabo previamente una valoración de los potenciales daños y riesgos individuales, dejando de lado sus intereses de investigación y teniendo el respeto debido de los derechos individuales básicos de la persona investigada.

\section{Investigación clínica no terapéutica en niños}

Hasta ahora se ha hablado de la investigación terapéutica que se lleva a cabo en pacientes que esperan obtener algunos beneficios de ella. Sin embargo, muchos proyectos de investigación son proyectos de investigación no terapéutica, en los cuales se sabe que la mayoría o todos los pacientes no obtendrán ningún beneficio personal.

Hasta el año 2004, en Alemania estaba prohibida la investigación no terapéutica en niños. Desde entonces está permitida, porque se ha seguido en esto las indicaciones de la Unión Europea de la buena práctica clínica, bajo el supuesto de que los riesgos y daños para los niños sean mínimos, el estudio no pueda ser llevado en adultos, exista un grupo que pueda beneficiarse en función de la edad o de la enfermedad, y el beneficio científico que se espera alcanzar sea alto.

Sucede, sin embargo, que la interpretación de lo que es "mínima molestia" o "mínimo riesgo", puede realizarse dentro de márgenes conceptuales muy amplios.

Tomemos un ejemplo: Para un estudio ortopédico de ligeras deformaciones de la articulación de la cadera, que en la edad infantil no provocan síntomas clínicos pero que son visibles a través de los rayos $\mathrm{X}$, se necesita una radiografía de la pelvis del niño, lo cual no le trae ningún beneficio. ¿Supone esta radiación adicional un riesgo mínimo para el niño? En Alemania se niega esta pregunta. Se podría, por ejemplo, utilizar otro procedimiento para este proyecto, por ejemplo la resonancia magnética, pero que está asociada a mayores costos. Mas aquí surge la pregunta de lo que se le puede demandar a una persona. Dado que los niños pequeños o especialmente angustiados pueden experimentar estados de ansiedad o incluso presentar cuadros de pánico con esta técnica, antes se les debería suministrar al niño un tranquilizante. Aunque en este último caso se puede discutir si hay o no daños mínimos, estos proyectos son aceptados recién desde el 2005. Antes se podía investigar con niños solo cuando existía la posibilidad de que la intervención 
investigatoria trajera algún beneficio para él. Esto es, a su propia salud.

La modificación de la ley de los medicamentos en Alemania en el año 2004 fue precedida por numerosos y encendidos debates, pues la investigación no terapéutica en niños solo se justifica cuando se destaca la utilidad para los futuros enfermos, y a esa utilidad se le concede gran valor. Con ese cambio de peso de una argumentación basada en los derechos a una que destaca el provecho para la comunidad va aparejada a la tendencia a minimizar determinados malestares y riesgos que pueden afectar a la persona en la investigación no terapéutica, debido a los futuros beneficios que se espera conseguir.

Otra argumentación que tiene raíces contractualistas pero que al mismo tiempo introduce el aspecto de justicia en la igualdad de oportunidades señala, algo cínicamente, que a los niños así como a las personas que no son capaces de tomar decisiones por sí solas debido a retardo mental no se les debe privar de la oportunidad de someterse de manera altruista a fines de investigación. Además, es de esperar en la investigación clínica frecuentemente un beneficio grupal para personas en la misma edad o con una enfermedad similar y, por lo tanto, se puede partir de una solidaridad de grupo.

Lo real es que no se puede presuponer la capacidad para una aprobación informada en pleno sentido en niños y en personas adultas con retardo mental. Ya solo la pregunta acerca de si perciben algo como un malestar ligero o un riesgo mínimo, querrían ellos mismos evaluarlas desde su perfecto derecho a un punto de vista subjetivo, independientemente del juicio de los médicos expertos. Además, un individuo capaz de tomar decisiones probablemente no permitirá que sean otros los que decidan cuándo y dónde debe él actuar altruistamente. Más bien, él querría hacerlo de acuerdo con sus criterios.

A la posibilidad de que un ser humano determine qué es el comportamiento altruista se opone el peligro de que personas que no desean ser parte de un experimento sean incluidas, solo porque extraños - aunque sean expertos- son del parecer de que los peligros y riesgos del sujeto experimental son necesarios, tomando en consideración los probables beneficios que otros pueden obtener.

\section{Investigación no terapéutica y aprobación informada de los adultos}

No solo en los niños sino también en los adultos la investigación no terapéutica puede ser controversial. Así, por ejemplo, un proyecto de investigación con el objetivo de averiguar cómo un paciente con cáncer al colon puede ser menos afectado por la enfermedad de altura sería relevante solo para pacientes futuros, que desean llevar a cabo un gran viaje a las montañas, probablemente en el extranjero. Los probandos mismos 
serían elegidos por su enfermedad de cáncer para el proyecto de investigación, y esta podría ocurrir en una cámara de presión. En un proyecto de esta índole estaría por tanto garantizado el cuidado de la vida de la persona, si el paciente antes ha sido minuciosamente examinado en materia de problemas coronarios y circulatorios. Pero los problemas vinculados a la escasa presencia de presión aérea no pueden de ninguna manera estar asociados a beneficio alguno. El proyecto de investigación solo se podría justificar por el beneficio que tendrían los futuros pacientes.

Un argumento utilitario como este es problemático porque los potenciales beneficios de los futuros pacientes con este proyecto no consisten en la mejoría de la terapia contra el cáncer, sino más bien en el que los pacientes de cáncer en el futuro puedan ser asesorados mejor en referencia a viajes a zonas elevadas.

Se podría argumentar que en este proyecto no terapéutico, las molestias, náuseas y dolores de cabeza solo son pasajeros y de ninguna manera arriesgan la vida y la salud de la persona, y que el paciente puede tomar por sí solo su decisión.

Pero, de otro lado, podría preguntarse si el beneficio que persigue el proyecto de investigación justifica las limitaciones del bienestar del paciente. Una argumentación simplemente contractualista en estos proyectos no formularía ninguna objeción. En una ar- gumentación utilitarista se podría discutir sobre el valor de la utilidad de los futuros pacientes en comparación con las molestias actuales de los pacientes de cáncer. En una argumentación basada en el derecho se podría investigar médicamente de un modo detenido si los pacientes de cáncer pueden ser sometidos a riesgos y malestares que no siempre son mínimos y, por lo tanto, dado que el tema no es urgente, lo adecuado es que no sean sujetos de un proyecto de esta índole.

\section{Preguntas éticas en la investigación con grupos placebo}

Finalmente, quiero presentar un ejemplo de por qué algunos diseños de investigación que contienen un grupo placebo son, desde el ángulo ético, problemáticos. Desde el punto de vista metodológico un diseño de investigación al azar, controlado y doble ciego a través de la presencia de un grupo placebo, concede un elevado nivel de significación, o permite emplear un número reducido de personas.

Esto significa que el proyecto de investigación puede desarrollarse durante un tiempo breve y por lo tanto originar menores costos. Además, está la regla de oro garantizada con sus resultados y su rigor metodológico. Desde el punto de vista metodológico y pragmático los diseños de investigación con un grupo placebo son también deseables. Sin embargo, desde el punto 
de vista ético las cosas se ven de otro modo.

No problemático es un grupo placebo para la supervisión de un nuevo tratamiento o de un nuevo medicamento, si los beneficios todavía no están asegurados o si los efectos colaterales no están suficientemente evaluados y no hay una terapia efectiva. En este caso, las personas experimentales o los pacientes que forman parte, por un procedimiento al azar, del grupo placebo, son un grupo control, los cuales ni están sometidos a un riesgo ni tampoco tienen que renunciar a una terapia efectiva.

Otro es el caso en la valoración ética, cuando, por ejemplo, un nuevo medicamento contra la depresión es comparado con uno ya evaluado, que se obtiene en el mercado, a fin de hallar si el nuevo medicamento es quizás más efectivo. Si se incorpora en este diseño un grupo de placebo en el cual pacientes depresivos permanecen sin ser tratados aunque el medicamento antidepresivo que funciona ya existe, entonces esto es desde el punto de vista ético problemático. Por un lado, porque un paciente depresivo y posiblemente suicida bajo determinadas circunstancias permanece sin ser tratado y, por otro, porque la pregunta de la efectividad del nuevo medicamento también se puede responder sin necesidad de recurrir a un grupo placebo, aunque con el costo de un menor nivel de significación.

Para los pacientes en el grupo placebo sería el proyecto probablemente un experimento no terapéutico; para los pacientes en los dos grupos de intervención un experimento terapéutico. Para los pacientes del grupo placebo el cuidado de su salud y de su vida no estarán en el primer lugar. Más bien para la conservación de un grupo placebo en este proyecto de investigación solo sería adecuada una argumentación utilitaria, la cual tiene que ver con el mayor nivel de conocimiento.

Igualmente problemáticos pueden ser los grupos placebo en la cirugía. Todavía en el año 2000 algunos médicos cirujanos se expresaron en Alemania en contra de la cirugía placebo (Uhl et al., 2000). En Estados Unidos se habían producido algunas intervenciones de esta naturaleza, en las cuales los pacientes solo en apariencia recibieron una intervención quirúrgica. Ellos fueron anestesiados para poder llevar a cabo un corte superficial, que les hacía creer que habían sido operados como otros pacientes. De esta manera se quería determinar si algunas intervenciones quirúrgicas son más efectivas que la ausencia de tratamiento $\mathrm{u}$ otras formas de tratamiento conservativo. En el marco de los esfuerzos por una medicina más basada en evidencias, de nuevo hay desde hace algunos años, también en Alemania, proyectos de investigación que contienen cirugía placebo. Ahora bien, es seguramente una cuestión de valoración si el paciente merece un pequeño malestar y el riesgo de una anestesia. 
Se podría señalar que es posible que a ellos se les ahorra un efecto dañino de mayor medida. Desde el punto de vista del cuidado del paciente esta valoración sería sin duda alguna justa. Sin embargo, surge aquí de nuevo la pregunta de las alternativas por evaluar antes de que un paciente por motivos de investigación sea sometido a riesgos perioperativos. ¿Por qué no se puede probar, como hasta ahora, una terapia quirúrgica contra una "espera calmada", u otro tratamiento convencional? El deseo de un diseño de investigación que se aproxime al ideal científico del experimento, y el esfuerzo de una medicina más basada en evidencias, constituyen sin duda la base para la modificación de la práctica de investigación en la cirugía. Así se manifestaron en Alemania el 2004 algunos reconocidos médicos a favor de la cirugía placebo (Seiler et al., 2004). Sin embargo, se muestra también en ese desarrollo que el criterio de la base en evidencias o el de la elevada significación de los resultados - ambos en realidad claves para la utilidad común y la ganancia de nuevos conocimientos que puede redundar en beneficio de los futuros pacientes - han ganado en significado. Por su parte, ha retrocedido la consideración hacia el probando individual y su realidad.

Evidentemente problemático desde el punto de vista del cuidado del paciente es un diseño quirúrgico placebo cuando, por ejemplo, en un derrame cerebral agudo, además del tratamiento medicamentoso se aplica una estimulación electrónica cerebral como tratamiento adicional, y esta es comparada con un grupo de pacientes que no solo recibe el tratamiento medicamentoso clásico sino, además, como en el caso del verdadero grupo de intervención, recibe una pequeña intervención quirúrgica cerebral, que debe simular una estimulación electrónica cerebral. Este grupo placebo recibe en verdad el tratamiento; es decir, no se le priva de ninguna terapia efectiva. Pero es sometido a un riesgo a través de la intervención microquirúrgica sin que pueda esperar beneficio terapéutico alguno. A esto se agrega que se trata, en el caso del grupo placebo, de una investigación no terapéutica en pacientes en los cuales, debido al agudo derrame cerebral padecido, no se puede dar por sentado que posean la capacidad para evaluar su situación y, consecuentemente, dar su aprobación informada al experimento al cual son sometidos.

A favor del tratamiento placebo habla el método objetivo que permite maximizar el nivel de significación de los resultados. Sin embargo, esta forma de microcirugía a la que se somete el grupo placebo esconde el riesgo - aunque pequeño - de un derrame cerebral o de una embolia.

Si se quiere dar pleno significado al cuidado del paciente debería procederse a llevar a cabo este diseño de investigación sin la forma de una intervención 
placebo. Entonces se necesitaría, para una comparación de la terapia estándar con una nueva, una más amplia forma de terapia sin grupo placebo, de un mayor número de pacientes para la misma significación estadística (y bajo condiciones también una más larga fase experimental) o se debería aceptar un menor nivel de significación.

\section{CONCLUSIÓN}

En la investigación médica en seres humanos en Alemania se observa la tendencia a que los derechos individuales de los probandos y de los pacientes en determinados casos son postergados (Graumann, 2007) en beneficio de un bien comunitario, sea en la forma de ganar conocimientos a través de la investigación básica o sea en la forma de beneficio para el grupo, o con otras palabras, en la forma de nuevas posibilidades terapéuticas para futuros pacientes o para beneficio de intereses individuales y colectivos de carrera.

Si se consideran las modificaciones producidas en el derecho y en las argumentaciones en los últimos años, por ejemplo que bajo determinadas condiciones niños y personas que no son capaces de tomar decisiones puedan ser incluidas en proyectos de investigación no terapéutica, es cierto que esto puede ser útil para otros seres humanos. Pero esto significa, asimis- mo, que personas cuyo estado de salud es particularmente frágil y que requieren cuidados especiales y particular dedicación, quedan sometidas a reflexiones de utilidad para el grupo. En esto se esconde el peligro de que personas que no pueden tomar decisiones por sí mismas y que tampoco pueden negarse, sean sometidas a decisiones dañinas tomadas por otros.

Paralelamente a la tendencia a debilitar el cuidado y los derechos de las personas que se encuentran en una posición frágil, se establece una cultura de la autonomía y de la aprobación informa$\mathrm{da}$, que tiende a enfatizar en exceso el derecho a la autodeterminación y, así, a descartar en buena medida cualquier otro criterio ético. En la investigación médica con seres humanos, el considerar solo el nivel de información del paciente, su capacidad para valorar las ventajas y desventajas personales y su disposición voluntaria a participar en experimentos, termina limitando su autonomía más que reforzándola. Pues cuando las personas sanas que deciden participar en un experimento y los pacientes no pueden confiar en que se ha llevado a cabo una valoración responsable del proyecto de investigación en el cual participan, la aprobación informada pierde su más importante fundamento: la confianza de que los otros han considerado todas las medidas de protección para ellas y que su bienestar es valorado y respetado. 


\section{REFERENCIAS}

Bundesdatenschutzgesetz del 1.1.1978 (última modificación del 5.2.2009).

Europäisches Parlament und der Rat der Europäischen Union (2001). Richtlinie zur Angleichung der Rechts-und Verwaltungsvorschriften der Mitgliedsstaaten über die Anwendung der guten klinischen Praxis bei der Durchführunf von klinischen Prüfungen mit Humanarzneimitteln

Europarat (1997). Übereinkommen zum Schutz der Menschenrechte und der Menschenwürde im Hinblick auf die Anwendung von Biologie und Medizin.

Gesetz über den Verkehr mit Arzneitmitteln (Arzneittelgesetz AMG). (2004). (12.AMG.Novelle).

Gesetz über Medizinprodukte (Medizinproduktegesetz -MPG) (2002).

Graumann, S. (2007). Freiwillige und informierte Einwilligung - ein forschungsethisches Auslaufmodell? Jahrbuch für Recht und Ethik, 15, 291-305.

Grundgesetz für die Bundesrepublik Deutschland. (1949) (I Die Grundrechte, en esp. art. 1 y art. 2).
Medical Research Council of Canada (1997). Code of Ethical Conduct for Research Involving Humans.

Mieth, D. (2002). Was wollen wir können? Ethik im Zeitalter der Biotechnologie. Herder-Verlag. Freiburg i. $\mathrm{Br}$.

Rothaar, M. (2006). Die EnqueteKommission Ethik und Recht der modernen Medizin des Deutsches Bundestages. Menshenrechtsmagazin, 2, 181-190.

Seiler, C. M.; Knaebel, H.P.; Wente, M. N.; Rothmund, M. \& Büchler, M. W. (2004). Plädoyer für mehr evidenzbasierte Chirugie. Deutsches Aerzteblatt, 6, 338-344.

Uhl, W.; Wente, M. N. \& Büchler, M. W. (2000). Chirurgisch-klinische Studien in der praktischen Durchführung. Der Chirurg, 71, 615-625.

Van Dijk, Y; van der Voort, P. H. J.; Kuiper, M. A. \& Kesecioglu, J. (2003). Research on subjects incapable of giving informed consent: the situation in Dutch intensive care departments. Intensive Care Medicine, 29, 2100-2101.

World Medical Association. (2008). Die Helsinki-Declaration: Ethical principles for medical research involving human subjects (version de Seúl). 